\title{
Clima motivacional percibido y motivación autodeterminada en Educación Física
}

Daniel López-Hernández ${ }^{1}$ y Carlos Fernández-Espínola²

1 Graduado en Educación Primaria, Mención en EF (Universidad de Huelva)

2 Universidad de Huelva

Email: ${ }^{2}$ carlos.fernandez@ddi.uhu.es

RESUMEN: LOS objetivos principales de este estudio fueron realizar un análisis descriptivo de variables motivacionales (clima motivacional percibido y tipos de motivación) en clases de EF, y analizar la relación existente entre estos factores. Asimismo, se examinó si había diferencias en función del género. Se utilizó una muestra de 55 estudiantes de sexto de Primaria pertenecientes a un centro de la provincia de Huelva. Los instrumentos empleados fueron el Cuestionario de Medida de las Estrategias Motivacionales en las clases de EF (CMEMEF) y Escala del Locus Percibido de Causalidad (PLOC) en EF. Los resultados mostraron puntuaciones medias más altas en el clima motivacional que implica a la tarea y los tipos de motivación más autodeterminados y una correlación significativa y positiva entre estas mismas variables. Por su parte, no se encontraron diferencias significativas en función del género. Finalmente, es destacable la influencia positiva que tiene sobre la motivación de los estudiantes, un clima transmitido por el docente en el que se valore el esfuerzo, la participación del alumnado, el progreso, la superación personal, etc.

PALABRAS CLAVE: Autodeterminación, Metas de Logro, Educación Primaria, Género.

\section{Perceived motivational climate and self-determined motivation in Physical Education}

ABSTRACT: The main purposes of this study were to perform a descriptive analysis of the motivational variables (perceived motivational climate and types of motivation) in PE classes, and the relationship between these factors. Furthermore, it was examined if there were differences according to gender. A sample of 55 students of sixth grade from a Primary School belonging to a center in the province of Huelva was used. The instruments used were the Questionnaire for Measuring Motivational Strategies in PE classes (CMEMEF) and Perceived Locus Scale Causality (PLOC) in PE. The findings shown mean scores higher in task-involving motivational climate and the self-determined forms of motivation. On the other hand, there were not significant differences based on gender. Finally, it is remarkable the positive influence it has on the motivation of the students, a climate transmitted by the teacher in which is valued the effort, the participation of the students, the progress, the personal improvement, etc.

KEY WORDS: Self-determination, Achievement Goals, Primary Education, Gender. 


\section{INTRODUCCIÓN}

Cada vez es más frecuente la ausencia de actividad física en niños y adolescentes, existiendo una alta tasa de abandono de práctica de actividad físicodeportiva entre los 12 y los 18 años (Chillón et al., 2009), especialmente en las chicas (Aibar, Bois, Generelo, Zaragoza, y Paillard, 2013). De hecho, Knisel, Opitz, Wossmann y Keteihuf (2009) destacaron la importancia de implementar en los centros educativos, programas de promoción de la salud que reduzcan el sedentarismo y aumenten los niveles de actividad física de los adolescentes, haciendo hincapié en el género femenino

En este sentido, en los últimos años ha cobrado un gran interés el estudio de factores motivacionales en el ámbito de la Educación Física (EF), (e. g. Chang, Chen, Tu, y Chi, 2016; Sánchez-Oliva, Pulido-González, Leo, González-Ponce, y GarcíaCalvo, 2017). Dicho interés se debe a que este constructo psicológico determina el comienzo, la prolongación en el tiempo y la finalización de los comportamientos humanos (González-Cutre, 2009). Este constructo permite conocer el esfuerzo que una persona invierte en conseguir unas metas determinadas, así como la perseverancia para lograrlas (Iso-Ahola y St. Clair, 2000)

La teoría de la autodeterminación (Deci y Ryan, 1985, 2000) y la teoría de metas de Logros (Nicholls, 1989) son las dos principales macroteorías que han permitido ahondar en el estudio de la motivación con estudiantes en las clases de EF.

El grado en el que las conductas humanas son realizadas por decisión propia, ha sido analizado en el campo de la EF por la teoría de la autodeterminación. Esta teoría establece diferentes formas de motivación a lo largo de un continuo; de mayor a menor nivel de autodeterminación encontramos: 1) motivación intrínseca, 2) motivación extrínseca compuesta por regulación integrada, regulación identificada, regulación introyectada y regulación externa; y finalmente 3) desmotivación (Ryan y Deci, 2000, Deci y Ryan, 2000). Esta teoría se basa en que las necesidades psicológicas básicas (competencia, autonomía, relación con los demás) influyen en la motivación del comportamiento humano. De hecho, la frustración de estas necesidades está asociada con una mayor motivación externa y desmotivación; y la satisfacción de las mismas con una mayor motivación intrínseca (Deci y Ryan, 2000). A su vez, la satisfacción o frustración de las necesidades psicológicas básicas está influenciada por el contexto social (Deci y Ryan, 1991). Por ello, es aquí donde juega un papel relevante la teoría de metas de logros, ya que uno de sus principales constructos es el clima motivacional transmitido, en este caso por el docente de EF (clima que implica a la tarea o clima que implica al ego). En concreto, un docente que transmite un clima ego es aquel que se centra en la comparación entre compañeros, la superación de los demás y únicamente en el resultado. Mientras que cuando un docente fomenta un clima que implique a la tarea, está valorando la superación personal, el esfuerzo y el proceso (Ames, 1992).

Asimismo, basándose en las estrategias TARGET de Ames (1992), Valentini, Rudisill y Goodway (1999) analizaron en mayor profundidad las ideas claves para que un docente genere un clima que implica a la tarea. Estas estrategias de forma breve son:

- Tarea: diseño de actividades variadas, retos personales, participación activa por parte de los alumnos y ayudar a estos a ser realistas con objetivos a corto plazo. 
- Autoridad: Involucrar a los alumnos en la toma de decisiones y otorgarles papeles de liderazgo. Asimismo, contribuir en su desarrollo de técnicas de autocontrol y autodirección.

- Reconocimiento: es preciso, reconocer el progreso personal y la mejora, asegurar la igualdad de oportunidades con respecto a las recompensas y ayudar en el refuerzo de la autoestima.

- Agrupación: los agrupamientos tienen que ser heterogéneos y flexibles, permitir la elección individual en la agrupación y ofrecer diferentes formas de agrupamiento.

- Evaluación: hay que involucrar al alumnado en la evaluación del progreso personal y el dominio de la tarea, así como proporcionar una retroalimentación, y una evaluación privada e individualizada.

- Tiempo: es necesario ofrecer oportunidades y tiempo suficientes para la mejora, contribuir a que el alumnado establezca sus propias tareas y la programación de estas.

De hecho, la teoría de la autodeterminación establece que el clima motivacional es un factor social que incide sobra la motivación mediante la satisfacción o frustración de las necesidades psicológicas mencionadas anteriormente (Deci y Ryan, 1985, 2000). En esta línea, diversos estudios han analizado la relación entre las necesidades psicológicas básicas y el clima motivacional en EF (Cox y Williams, 2008; MéndezGiménez, Fernández-Río, y Cecchini-Estrada, 2013; Sevil, Abós, Aibar, Julián, y García-González, 2015).

En definitiva, la literatura científica ha demostrado las relaciones existentes entre el clima motivacional transmitido por el docente y los efectos que se producen en la motivación de los alumnos. Por ello, con el fin de corroborar los estudios ya existentes, los objetivos principales de este estudio fueron realizar un análisis descriptivo de factores motivacionales en las clases de EF, y analizar las relaciones entre el clima motivacional transmitido por el docente de EF y los diferentes tipos de motivación. Asimismo, teniendo en cuenta que los niveles de práctica disminuyen en la adolescencia, y especialmente, en el género femenino (Martínez-Baena et al., 2012), en este estudio se planteó como objetivo secundario: examinar la influencia del género en las variables motivacionales estudiadas. En relación con la literatura científica revisada, se teorizó que, si el clima motivacional que transmitía el docente implicaba a la tarea, el alumnado presentaría puntuaciones altas en las formas más autodeterminadas de motivación. Además, se propuso como hipótesis que los chicos presentarían mayores niveles de motivación que las chicas en las clases de EF.

\section{MÉTODO}

\subsection{Diseño}

El presente trabajo tiene un diseño transversal, con estrategia descriptiva y un muestreo intencional (Ato, López, y Benavente, 2013).

\subsection{Participantes}

La muestra de este estudio estuvo compuesta por un total de 55 estudiantes (27 chicos y 28 chicas) pertenecientes a un Centro de Educación Primaria de la provincia de Huelva. La edad de los participantes del estudió estaba comprendida 
entre los 11 y los 14 años $(M=12.27$ y $D T=.52)$. La participación por parte del alumnado en el estudio fue optativa y la cumplimentación de los cuestionarios se realizó durante las clases de EF.

\subsection{Instrumentos}

Cuestionario de Medida de las Estrategias Motivacionales en las clases de Educación Física (CMEMEF). Se usó la versión diseñada y validada por Cervelló, Moreno, del Villar y Reina (2007), basada en el modelo de intervención motivacional propuesto por Ames (1992). Este cuestionario constaba de 24 ítems agrupados en dos dimensiones (12 ítems por dimensión), que son el clima motivacional que implica a la tarea (e.g. "Nos animan a que nos ayudemos entre compañeros durante las tareas") y el clima motivacional que implica el ego (e.g. "Solo se evalúa el resultado final, sin importarle si progreso respecto a cómo lo hacía antes"). La escala va encabezada por la sentencia "En las clases de Educación Física...", respondiéndose mediante una escala tipo Likert que va de 0 (Totalmente en desacuerdo) a 10 (Totalmente de acuerdo). La fiabilidad de esta escala fue de .77 para el clima tarea y de .70 para el clima ego.

Escala del Locus Percibido de Causalidad en Educación Física. Se utilizó la versión testada en el contexto español por Moreno, González-Cutre y Chillón (2009) de la Perceived Locus of Causality Scale (PLOC) de Goudas, Biddle y Fox (1994). La sentencia previa a los ítems fue "Participo en las clases de Educación Física". La escala estaba compuesta por 24 ítems agrupados en cuatro dimensiones, compuesta cada una por cuatro ítems. Las dimensiones miden la Motivación Intrínseca (e.g. "Porque la educación física es divertida"), Regulación Identificada (e.g. "Porque quiero aprender habilidades deportivas"), Regulación Introyectada (e.g. "Porque me sentiría mal conmigo mismo si no lo hiciera"), Regulación Externa (e.g. "Porque tendré problemas si no lo hago") y Desmotivación (e.g. "Pero no comprendo porque debemos tener educación física"). Las respuestas fueron puntuadas con una escala tipo Likert, con un rango de puntuación que oscilaba entre 1 (Totalmente en desacuerdo) y 7 (Totalmente de acuerdo). Se obtuvieron valores de consistencia interna de .69 para la motivación intrínseca, .72 para la regulación identificada, .48 para la regulación introyectada, .49 para la regulación externa y .70 para la desmotivación.

\subsection{Procedimiento}

En primer lugar, se contactó con el equipo directivo de los centros educativos para comunicarles los objetivos del estudio y solicitar su colaboración. Posteriormente, se concertó con el tutor una fecha para la administración de los cuestionarios y se solicitó alumnado una autorización por escrito de sus padres o tutores legales para participar en el estudio, debido a su minoría de edad. Finalmente, se llevó a cabo la administración de los cuestionarios, estando presente el investigador principal, con la intención de explicar brevemente el objetivo del estudio e informar de cómo cumplimentar el cuestionario adecuadamente, así como solventar las posibles dudas que puedan surgir durante la realización del mismo. Además, se comunicó a los participantes que la cumplimentación del cuestionario tendría una duración de unos 15 minutos aproximadamente y que sus respuestas serían recogidas de forma anónimas.

\subsection{Análisis de datos}

Se empleó el programa estadístico SPSS para el análisis de los diferentes tipos de variables. En primer lugar, se depuro la matriz de datos. Posteriormente, se calcularon los resultados estadísticos descriptivos de todas las variables objeto de estudio (medias y desviaciones típicas, asimetría y curtosis), se analizó la consistencia 
interna de cada factor mediante el coeficiente de Alfa de Cronbach. Asimismo, se analizó la normalidad de los datos, observando si los valores de curtosis y asimetría de las variables estudiadas oscilaban entre 1.00 y -1.00 , lo cual corresponde a una distribución normal de los datos (George y Mallery, 2001). A continuación, se realizó la correlación de los climas y los diferentes tipos de motivación para averiguar las relaciones entre las diferentes variables. Finalmente, se realizó una prueba $T$ de Student para averiguar si existían diferencias en función del género entre las variables del estudio.

\section{RESULTADOS}

\subsection{Estadísticos descriptivos}

En la Tabla 1 se muestran los estadísticos descriptivos de cada una de las variables de estudio. Teniendo en cuenta que la respuesta sobre el clima motivacional percibido oscilaba entre 0 y 10 y las respuestas de los diferentes tipos de motivación variaban entre 1 a 7 , destacamos: que la puntuación media del clima motivacional que implica a la tarea fue mayor $(M=6.62)$ a la puntuación media del clima ego $(M=3.46)$. En lo que respecta a los tipos de motivación, las puntuaciones medias más altas correspondieron a las formas más autodeterminadas. En concreto, la puntuación más alta fue para la regulación identificada, con un valor de 5.90; seguida de la motivación intrínseca $(M=5.58)$. En cambio, la puntuación media más baja correspondió a la desmotivación $(M=3.04)$. En lo que respecta a la distribución de los datos, los valores de asimetría y curtosis de todas las variables oscilaron entre 1.00 y -1.00 (ver tabla 1), por lo que se cumplieron los supuestos de normalidad.

Tabla 1. Estadísticos descriptivos con respecto al clima motivacional y los tipos de motivación

\begin{tabular}{lccccc}
\hline & $\mathbf{N}$ & $\boldsymbol{M}$ & $\boldsymbol{D T}$ & $\mathbf{A}$ & $\mathbf{C}$ \\
\hline Clima Tarea & 55 & 6.62 & 1.44 & -.74 & -.01 \\
Clima Ego & 55 & 3.46 & 1.47 & .21 & -.67 \\
$\begin{array}{l}\text { Motivación } \\
\text { Intrínseca }\end{array}$ & 55 & 5.58 & 1.28 & -1.00 & .67 \\
$\begin{array}{l}\text { Regulación } \\
\text { Identificada }\end{array}$ & 55 & 5.90 & 1.13 & -.88 & -.41 \\
$\begin{array}{l}\text { Regulación } \\
\text { Introyectada }\end{array}$ & 55 & 4.42 & 1.26 & .32 & -.30 \\
$\begin{array}{l}\text { Regulación Externa } \\
\text { Desmotivación }\end{array}$ & 55 & 3.93 & 1.24 & .32 & -.71 \\
Nota: N = Número de sujetos; $M=$ Media; $D T=$ Desviación tí́pica; A = Asimetría; C = Curtosis.
\end{tabular}

\subsection{Correlaciones bivariadas}

En el análisis de correlación entre las variables del estudio (ver Tabla 2), se obtuvo que el clima que implica a la tarea se correlacionó, por un lado, positiva y significativamente con la motivación intrínseca, la regulación Identificada y la regulación introyectada, y, por otro lado, de forma negativa y estadísticamente significativa con el clima ego. En cambio, el clima motivacional que implica al ego 
correlacionaba de manera positiva y significativa con la desmotivación de los estudiantes de EF. Asimismo, el clima ego mostró relaciones negativas y significativas con la motivación intrínseca y la regulación identificada.

En cuanto a los diferentes tipos de motivación, el análisis de correlación mostró que la motivación intrínseca se relacionó positiva y significativamente con la regulación identificada y la regulación introyectada, y negativa y significativamente, con la desmotivación. Por su parte, la regulación identificada presentó correlaciones similares a la motivación intrínseca. Finalmente, la regulación externa mostró una relación positiva y significativa con la desmotivación.

Tabla 2. Correlación de bivariadas entre las variables del estudio

\begin{tabular}{|c|c|c|c|c|c|c|c|}
\hline Variables & 1 & 2 & 3 & 4 & 5 & 6 & 7 \\
\hline 1. Clima Tarea & - & $-.47^{* *}$ & $.56^{\star \star}$ & $.55^{\star *}$ & $.40^{* *}$ & .01 & -.22 \\
\hline 2.Clima Ego & - & - & $-.28^{*}$ & $-.30^{*}$ & -.06 & .18 & $.29^{*}$ \\
\hline 3.Motivación Intrínseca & - & - & - & $.82^{\star *}$ & $.43^{\star *}$ & -.13 & $-.48^{* *}$ \\
\hline 4.Regulación Identificada & - & - & - & - & $.40^{* *}$ & -.21 & $-.49^{* *}$ \\
\hline 5.Regulación Introyectada & - & - & - & - & - & .24 & .01 \\
\hline $\begin{array}{l}\text { 6.Regulación Externa } \\
\text { 7.Desmotivación }\end{array}$ & - & - & - & - & - & - & \\
\hline
\end{tabular}

\subsection{Diferencia de medias en función del género}

En la Tabla 3, se presentan las diferencias de medias en función del género. De forma general, las chicas presentaron valores ligeramente más altos tanto en el clima tarea $(M=6.73)$ como en el clima ego $(M=3.54)$. En lo que respecta a los tipos de motivación, las chicas mostraron puntuaciones superiores en los tipos de motivación más autodeterminados, concretamente, en la motivación intrínseca $(M=$ $5.73)$ y regulación identificada $(M=6.04)$. En cambio, los chicos presentaron una mayor desmotivación $(M=3.35)$. En todas las variables, los valores de significación obtenidos fueron mayores a .05, por lo que en ambos casos se aceptó la hipótesis nula y se asumió que no había diferencias significativas entre chicos y chicas en ninguna de las variables estudiadas.

Tabla 3. Diferencias de medias en función del género

\begin{tabular}{llllll}
\hline \multirow{2}{*}{ Clima Tarea } & Sexo & $\mathrm{N}$ & $M$ & $D M$ & $D T$ \\
\hline \multirow{2}{*}{ Clima Ego } & Hombre & 27 & 6.52 & -.21 & 1.49 \\
& Mujer & 28 & 6.73 & & 1.42 \\
\hline \multirow{2}{*}{ Motivación Intrínseca } & Hombre & 27 & 3.38 & \multirow{2}{*}{-.16} & 1.47 \\
& Mujer & 28 & 3.54 & & 1.59 \\
\hline Regulación Identificada & Hombre & 27 & 5.42 & \multirow{2}{*}{-.31} & 1.30 \\
& Mujer & 28 & 5.73 & & 1.28 \\
\hline
\end{tabular}




\begin{tabular}{llllll} 
& Mujer & 28 & 6.04 & & 1.08 \\
\hline \multirow{2}{*}{ Regulación Introyectada } & Hombre & 27 & 4.26 & \multirow{2}{*}{-.32} & 1.17 \\
& Mujer & 28 & 4.58 & & 1.34 \\
\hline \multirow{2}{*}{ Regulación Externa } & Hombre & 27 & 3.91 & \multirow{2}{*}{-.05} & 1.40 \\
& Mujer & 28 & 3.96 & & 1.10 \\
\hline \multirow{2}{*}{ Desmotivación } & Hombre & 27 & 3.35 & \multirow{2}{*}{.61} & 1.40 \\
& Mujer & 28 & 2.74 & & 1.55 \\
\hline
\end{tabular}

Nota: $\mathrm{N}=$ Número de sujetos; $M=$ Media; $D M=$ Diferencia de Medias; $D T$ = Desviación típica.

\section{DISCUSIÓN}

Uno de los dos objetivos principales de este estudio fue realizar un análisis descriptivo de factores motivacionales en clases de EF de estudiantes de Educación Primaria. En este sentido, en lo que respecta a los climas motivacionales, las puntuaciones medias obtenidas en el clima que implica a la tarea fueron superiores al clima que implica al ego. Resultados que coinciden con otros estudios que analizaron estas variables en el contexto educativo de EF en Primaria (Buzón y Conde, 2017; Gutiérrez y López, 2012) y en Secundaria (Cera, Almagro, Conde, y Sáenz-López, 2015; Sevil, Aibar, Abós, y García-González, 2017).

En cuanto a las diferentes formas de motivación, las puntuaciones medias más altas se obtuvieron en los tipos más autodeterminados (motivación intrínseca y regulación identificada), mientras que la puntuación más baja correspondió a la desmotivación. Resultados que están en la línea con los mostrados por Cera et al. (2015), Méndez-Giménez, Fernández-Río y Cecchini-Estrada (2016), y Sevil et al. (2017).

El otro propósito principal del estudio fue analizar las relaciones entre el clima motivacional percibido y las diferentes formas de motivación. El análisis de correlación mostró una asociación positiva y significativa entre el clima que implica a la tarea y los tipos de motivación más autodeterminados, y una relación negativa y significativa con la desmotivación, coincidiendo con el estudio de Cera et al. (2015), en el que se obtuvieron valores similares, aunque únicamente fueron significativos los valores de correlación positivos. Esta asociación positiva y significativa entre el clima tarea y la motivación intrínseca en las clases de EF, también fue hallada por Moreno, Jiménez, Gil, Aspano y Torrero (2011). Por otro lado, en lo que respecta al clima que implica al ego, en este trabajo, correlacionó positiva y significativamente con la desmotivación de los estudiantes en las clases de EF, y se halló una relación negativa y significativa con la motivación intrínseca y la regulación identificada. En la misma línea, en el estudio realizado por Cera et al. (2015), el clima ego también se relacionó de forma positiva y significativa con la desmotivación de los alumnos en EF. En este sentido, el clima tarea influye positivamente en los tipos de motivación más autodeterminados, y el clima ego influye positivamente en la desmotivación. De hecho, la teoría de la autodeterminación (Deci y Ryan, 1985, 2000), establece que el contexto social (en este caso el clima motivacional transmitido por el docente), va a influir en la satisfacción o frustración de las necesidades psicológicas básicas, y a su vez éstas incidirán de forma positiva o negativa en la motivación de los estudiantes en las clases de EF. Esta afirmación ha sido testada en diversos estudios cuasi-experimentales (eg. González-Cutre, Sicilia, y Moreno, 2011; Sevil, Julián, Abarca-Sos, Aibar, y GarcíaGonzález, 2014), en los que tras una intervención en el que se emplearon una serie de 
estrategias para generar un clima orientado a la tarea (por ejemplo, con la presencia de tareas novedosas, participación activa del alumnado en la toma de decisiones y una evaluación del progreso personalizada y privada, entre otras) obtuvieron aumentaron los niveles de motivación más autodeterminados y redujeron la desmotivación.

En lo que respecta a la diferencia de medias en función del género. Contrario a nuestra hipótesis, en este estudio, las chicas presentaron valores superiores en los tipos de motivación más autoderminados y valores inferiores en la desmotivación. Sin embargo, estos resultados no fueron significativos. En esta línea, se encontraron resultados similares (aunque este caso, sí fueron significativos) en el estudio de Sevil, Abós, Julián, Murillo y García-González (2015), en los que las chicas presentaron una mayor motivación intrínseca y menor desmotivación que los chicos durante una unidad didáctica sobre expresión corporal.

Por último, es reseñable que el presente estudio tiene algunas limitaciones. Entre ellas, cabe destacar la escasez del tamaño muestral, ya que únicamente se ha realizado en un centro educativo. Por otra parte, la consistencia interna de algunas dimensiones (motivación intrínseca, regulación identificada y regulación introyectada) no presentó valores aceptables de fiabilidad $(p \geq .70)$. Además, las diferencias de medias de las variables motivacionales en función del género no presentaron valores significativos.

\section{CONCLUSIONES}

Los resultados del análisis descriptivo mostraron que los alumnos percibían un clima que implica a la tarea por parte del docente de EF, presentando además puntuaciones más altas en los tipos de motivacionales autodeterminadas.

Por su parte, de acuerdo con la literatura científica, el clima tarea correlacionó de forma positiva y significativa con la motivación intrínseca y la regulación identificada, y de forma negativa con la desmotivación. Por tanto, teniendo en cuenta los resultados de este estudio y en sintonía con lo revisado en la literatura, parece conveniente apoyar un clima en el que se valora el esfuerzo, la participación del alumnado, el progreso y la superación personal (clima tarea), ya que éste, podría influir en el aumento de los niveles de motivación intrínseca y en la disminución de la desmotivación sobre el alumnado en la asignatura de EF.

Finalmente, no se encontraron diferencias significativas entre chicos y chicas en ninguna de las variables motivacionales estudiadas, por lo que son necesarios otros estudios que profundicen en este asunto.

\section{REFERENCIAS}

Aibar, A., Bois, J. E., Generelo, E., Zaragoza Casterad, J., y Paillard, T. (2013). A cross-cultural study of adolescents' physical activity levels in France and Spain. European Journal of Sport Science, 13(5), 551-558.

Ames, C. (1992). Achievement goals, motivational climate, and motivational processes. En G.C. Roberts (Ed.), Motivation in sport and exercise (pp. 161-176). Champaign, IL: Human Kinetics. 
Ato, M., López, J. J., y Benavente, A. (2013). Un sistema de clasificación de los diseños de investigación en psicología. Anales de Psicología, 29(3), 10381059.

Buzón, P., y Conde, C. (2017). Análisis de las relaciones entre los climas motivacionales y las necesidades psicológicas básicas en Educación Física. Emotion: Revista de Educación, Motricidad e Investigación, 9, 3-12.

Cera, E., Almagro, B. J., Conde, C., y Sáenz-López, P. (2015). Inteligencia emocional y motivación en educación física en secundaria. Retos, 27, 8-13.

Cervelló, E., Moreno, J. A., del Villar, F., y Reina, R. (2007). Desarrollo y validación de un instrumento de medida de las estrategias motivacionales empleadas en las clases de educación física. Revista Iberoamericana de Psicología del Ejercicio y el Deporte, 2(2), 53-72.

Cox, A., y Williams, L. (2008). The roles of perceived teacher support, motivational climate, and psychological need satisfaction in students' physical education motivation. Journal of Sport and Exercise Psychology, 30(2), 222-239.

Chang, Y. K., Chen, S., Tu, K. W., y Chi, L. K. (2016). Effect of autonomy support on self-determined motivation in elementary physical education. Journal of sports science \& medicine, 15(3), 460-466.

Chillón, P., Ortega, F., Ruiz, J., Pérez, I., Martín-Matillas, M., Valtueña, J., ...Delgado, M. (2009). Socio-economic factors and active commuting to school in urban Spanish adolescents: the AVENA study. European Journal of Public Health, 19 (5), 470-476.

Deci, E. L., y Ryan, R. M. (1985). Intrinsic motivation and self-determination in human behavior. New York: Plenum.

Deci, E. L., y Ryan, R. M. (1991). A motivational approach to self: Integration in personality. En R. Dienstbier (Ed.), Nebraska symposium on motivation: Vol. 38. Perspectives on motivation (pp. 237-288). Lincoln, NE: University of Nebraska Press.

Deci, E. L., y Ryan, R. M. (2000). The "what" and "why" of goal pursuits: Human needs and the selfdetermination of behavior. Psychological Inquiry, 11, 227-268.

George, D., y Mailery, M. (2001). Using SPSS for Windows step by step: a simple guide and reference. Boston, MA: Aliyn \& Bacon

González-Cutre, D. (2009). Motivación, creencias implícitas de habilidad, competencia percibida y flow disposicional en clases de educación física. Tesis Doctoral. Universidad de Almería. Almería.

González-Cutre, D., Sicilia, A., y Moreno, J.A. (2011). Un estudio cuasi-experimental de los efectos del clima motivacional tarea en las clases de educación física. Revista de Educación, 356, 677-700.

Goudas, M., Biddle, S., y Fox, K. (1994). Perceived locus of causality, goal orientations, and perceived competence in school physical education classes. British Journal of Educational Psychology, 64(3), 453-463. 
Gutiérrez, M., y López, E. (2012). Clima motivacional, razones para la disciplina y comportamiento en Educación Física. Revista Internacional de Medicina y Ciencias de la Actividad Física y el Deporte, 12(46), 235-251.

Iso-Ahola, S. E., y St. Clair, B. (2000). Toward a theory of exercise motivation. Quest, $52,131-147$.

Knisel, E., Opitz, S., Wossmann, M., y Keteihuf, K. (2009). Sport motivation and physical activity of students in three European schools. International Journal of Physical Education, 46, 40-53.

Martínez-Baena, A. C., Chillón, P., Martín-Matillas, M., Pérez López, I., Castillo, R., Zapatera, B., ... y Tercedor, P. (2012). Motivos de abandono y no práctica de actividad físico-deportiva en adolescentes españoles: estudio Avena. Cuadernos de Psicología del Deporte, 12(1), 45-54.

Méndez-Giménez, A., Fernández-Río, J., y Cecchini-Estrada, J. A. (2013). Climas motivacionales, necesidades, motivación y resultados en Educación Física. Aula abierta, 41(1), 63-72.

Méndez-Giménez, A., Fernández-Río, J., y Cecchini-Estrada, J. A. (2016). Vallerand's model in asturian adolescents: implementation and development. Revista Internacional de Medicina y Ciencias de la Actividad Física y del Deporte, 16(64), 703-721. doi:10.15366/rimcafd2016.64.006

Moreno, B., Jiménez, R., Gil, A., Aspano, M. I., y Torrero, F. (2011). Análisis de la percepción del clima motivacional, necesidades psicológicas básicas, motivación autodeterminada y conductas de disciplina de estudiantes adolescentes en las clases de educación física. Motricidad. European Journal of Human Movement, 26, 1-24.

Moreno, J. A., González-Cutre, D., y Chillón, M. (2009). Preliminary validation in Spanish of a scale designed to measure motivation in physical education classes: the Perceived Locus of Causality (PLOC) Scale. The Spanish Journal of Psychology, 12(1), 327-337.

Nicholls, J. G. (1989). The competitive ethos and democratic education. Cambridge, Massachusetts: Harvard University Press.

Ryan, R. M., y Deci, E. L. (2000). Self-determination theory and the facilitation of intrinsic motivation, social development and well-being. American Psychologist, 55(1), 68-78.

Sánchez-Oliva, D., Pulido-González, J. J., Leo, F. M., González-Ponce, I., y GarcíaCalvo, T. (2017). Effects of an intervention with teachers in the physical education context: A Self-Determination Theory approach. PLOS ONE, 12(12), e0189986. doi: 10.1371/journal.pone.0189986

Sevil, J., Abós, Á., Aibar, A., Julián, J. A., y García-González, L. (2015). Gender and corporal expression activity in physical education. European Physical Education Review, 22(3), 372-389. doi:10.1177/1356336x15613463

Sevil, J., Abós, Á., Julián, J. A., Murillo, B., y García-González, L. (2015). Género y motivación situacional en Educación Física: claves para el desarrollo de 
estrategias de intervención. Revista Internacional de Ciencias del Deporte, 11(41), 281-296.

Sevil, J., Aibar, A., Abós, Á., y García González, L. (2017). El clima motivacional del docente de Educación Física: ¿Puede afectar a las calificaciones del alumnado? Retos, 31, 98-102.

Sevil, J., Julián, J. A., Abarca-Sos, A., Aibar, A., y García-González, L. (2014). Efecto de una intervención docente para la mejora de variables motivacionales situacionales en Educación Física. Retos, 26, 108-113.

Valentini, N. C., Rudisill, M. E., y Goodway, J. D. (1999). Incorporating a mastery climate into physical education: It's developmentally appropriate! Journal of Physical Education, Recreation and Dance, 70(7), 28-61. 